\title{
Index C-reactive protein predicts increased severity in acute sigmoid diverticulitis
}

This article was published in the following Dove Press journal:

Therapeutics and Clinical Risk Management

\author{
Aristotelis Kechagias ${ }^{1,2}$ \\ Anastasios Sofianidis' \\ Georgios Zografos ${ }^{3}$ \\ Emmanouel Leandros ${ }^{3}$ \\ Nicholas Alexakis ${ }^{3}$ \\ Christos Dervenis' \\ 'Department of Surgery, \\ Konstantopouleion Hospital, \\ Athens, Greece; ${ }^{2}$ Department of \\ Gastrointestinal Surgery, Kanta- \\ Häme Central Hospital, Hämeenlinna, \\ Finland; ${ }^{3}$ First Department of \\ Propaedeutic Surgery, Hippocratio \\ Hospital, National and Kapodistrian \\ University of Athens, Athens, Greece
}

Correspondence: Aristotelis Kechagias

Department of Surgery,

Konstantopouleion Hospital, Agias

Olgas 3-5 Street, 14233 Nea lonia,

Athens, Greece

Tel +302132057904

Email aristotelis.kechagias@yahoo.gr
Purpose: Conservative management is successful in unperforated (Hinchey Ia) acute diverticulitis (AD) and also generally in local perforation or small abscesses (Hinchey $\mathrm{Ib}$ ). A higher degree of radiological severity (Hinchey $>\mathrm{Ib}$ ), ie, a larger abscess $(>3-4 \mathrm{~cm}$ ) or peritonitis, commonly requires percutaneous drainage or surgery. Retrospective studies show that high levels of C-reactive protein (CRP) distinguish Hinchey Ia from all cases of minor and major perforations (Hinchey $>$ Ia). The current study aims to evaluate the usefulness of CRP in distinguishing $\mathrm{AD}$ with a higher degree of severity (Hinchey $>\mathrm{Ib}$ ) from cases that can be treated noninvasively (Hinchey Ia/Ib).

Methods: Data from consecutive patients with AD were collected prospectively. All underwent computed tomography (CT). Index parameters obtained at the initial evaluation at the emergency unit were analyzed to assess the association with the outcome. The exclusion criteria comprised concomitant conditions that affected CRP baseline levels.

Results: Ninety-nine patients were analyzed. Eighty-eight had mild radiological grading (Hinchey $\mathrm{Ia} / \mathrm{Ib}$ ), and 11 had severe radiological grading (Hinchey $>\mathrm{Ib}$ ) (median index CRP $80 \mathrm{mg} / \mathrm{L}$ vs $236 \mathrm{mg} / \mathrm{L}[P<0.001]$ ). White blood cells, neutrophils/lymphocytes, serum creatinine, serum glucose, generalized peritonitis, generalized abdominal tenderness, urinary symptoms, and index CRP were related to severe disease. Index CRP was the only independent predictor for Hinchey $>\operatorname{Ib}(P=0.038)$. The optimal cutoff value calculated by receiver operating characteristic curve analysis was found to be $173 \mathrm{mg} / \mathrm{L}$ (sensitivity $90.9 \%$, specificity $90.9 \%, P<0.001$ ). All patients who underwent radiological drainage or surgery had an index CRP $>173 \mathrm{mg} / \mathrm{L}$ and Hinchey $>\mathrm{Ib}$.

Conclusion: CRP levels $>173 \mathrm{mg} / \mathrm{L}$ obtained at the initial evaluation at the emergency unit predict major acute complications in $\mathrm{AD}$. These patients commonly require urgent percutaneous drainage or surgical management.

Keywords: acute diverticulitis, C-reactive protein, severity prediction, computed tomography

\section{Introduction}

Acute diverticulitis (AD) of the colon is an increasingly prevalent condition in Western societies. ${ }^{1,2}$ In the US, a 50\% rise in incidence was documented for the 2000-2007 period compared to the 1990s. ${ }^{1}$ Similarly, a 2.8 -fold increase in the hospital admission rate has been reported in northern Europe over a period of 25 years, contributing to a substantial economic burden. ${ }^{2}$

Patients with AD experience a varying degree of disease severity. Approximately $80 \%-85 \%$ of patients have a clinically mild disease and are treated conservatively, ${ }^{1-3}$ even as outpatients. ${ }^{4}$ Nonoperative treatment is successful in cases of the phlegmonous disease ${ }^{4}$ however, recent data have shown that this is also the rule for those with radiological signs of local perforation or a small abscess. ${ }^{4-9}$ On the contrary, patients with a 
larger abscess $(>4 \mathrm{~cm})$ commonly necessitate percutaneous drainage or operative management, whereas frank peritonitis - either purulent or fecal - mandates an emergency operation. ${ }^{10}$ Currently, there is a trend toward less operative and interventionist radiological treatment in selected patients with an absence of sepsis or significant comorbidities, regardless of the imaging findings. ${ }^{4-6,8,10,11}$ Computed tomography (CT) is the preferred imaging method for grading the severity of AD. ${ }^{10}$ However, it should be emphasized that judgment for the actual necessity of invasive treatment should be based on both clinical and radiological grounds, as CT severity alone does not necessarily mandate the need for drainage or an operation. ${ }^{5,6,8,11}$

C-reactive protein (CRP) has been found to be a useful marker in the prediction of the inflammation grade and severity of AD. ${ }^{10}$ According to retrospective data that assessed imaging severity, CRP may discriminate the mere phlegmonous disease from the remainder of cases with either minor or major perforation (local or diffuse free intraperitoneal air, a small or large abscess, peritonitis). ${ }^{3,12-14}$ However, the value of CRP in predicting the severity of AD should be further evaluated according to specific clinical problems, since patients with a contained perforation or small abscess are as a rule treated conservatively during the acute episode. ${ }^{4-9}$

The present observational study uses prospectively collected data to evaluate the role of index CRP in predicting the increased severity of $\mathrm{AD}$, defined as the presence of a larger abscess $(>3 \mathrm{~cm})$ or peritonitis.

\section{Methods}

Demographic, clinical, laboratory, and imaging data were collected prospectively from all patients treated for AD at the Department of Surgery, Konstantopouleion Hospital, Athens, Greece, between 2011 and 2014. The study was approved by the ethics committee of the hospital (local authorization number 19183, research registry identification number 2575).
All patients provided written informed consent to participate in the study. The management policy of AD was based on the recommendation available during the period of the study (subsequently updated). ${ }^{15}$ All patients had a CT scan, which was performed during the initial evaluation at the emergency unit. Intravenous contrast was used for the CT scans unless contraindicated. The modified Hinchey classification by Wasvary et $\mathrm{al}^{28}$ was used to grade radiological severity (Table 1). ${ }^{10}$ Antibiotics were routinely administered. Patients with peritonitis and severe sepsis underwent an emergency operation. Percutaneous drainage was performed on all patients with an abscess $>5 \mathrm{~cm}$. Patients with a smaller abscess and those with a local perforation (local extracolonic air) or the simple phlegmonous disease were treated conservatively. The patients' clinical status was assessed frequently, as deterioration is an indicator of the failure of conservative therapy. Blood cultures were obtained in the case of clinical signs of sepsis. A follow-up colonoscopy was performed for all patients.

We analyzed the laboratory and clinical parameters obtained at the initial evaluation of the patient at the emergency unit (index parameters). They were correlated with the level of imaging severity expressed by the CT findings. Patients with the phlegmonous disease (Hinchey Ia) or with a contained perforation or small local abscess (Hinchey Ib) were classified as having mild radiological disease. Patients with a distant or larger abscess $(>4-5 \mathrm{~cm})$ or frank peritonitis were classified as having severe radiological disease (Hinchey $>\mathrm{Ib}$ ).

Dedicated inclusion criteria were employed, as CRP baseline levels may be affected by various factors. ${ }^{16}$ Liver failure and therapies or diseases affecting the acute-phase stimulus are common causes for the muted or reduced reaction of CRP to infection (Table 2). ${ }^{16}$ Index CRP levels during AD may be reduced in such patients with the same degree of radiological severity. On the other hand, concomitant infections, tissue necrosis or trauma, neoplasia, and other inflammatory

Table I Depiction of treatment and radiological severity according to the modified Hinchey classification by Wasvary et al ${ }^{28}$

\begin{tabular}{llll}
\hline $\begin{array}{l}\text { Modified Hinchey } \\
\text { grading (N=99) }\end{array}$ & Findings in CT scan & $\begin{array}{l}\text { CT-guided } \\
\text { drainage (n=2) }\end{array}$ & Operations (n=7) \\
\hline Ia (7I) & Phlegmon (absence of perforation) & 0 & 0 \\
Ib (I7) & Localized free air or confined pericolic abscess & 0 & 0 \\
II (7) & Pelvic, distant intra-abdominal, or retroperitoneal abscess & 2 & 3 laparoscopic lavages \\
III (2) & Generalized purulent peritonitis & 0 & I laparotomy and lavage \\
IV (2) & Fecal peritonitis & 0 & I Hartmann sigmoidectomy \\
& & & I Hartmann sigmoidectomy \\
I sigmoidectomy with anastomosis \\
\end{tabular}

Abbreviation: CT, computed tomography. 
Table 2 Exclusion criteria (conditions potentially affecting CRP basal levels)

\begin{tabular}{ll}
\hline Exclusion criterion & $\begin{array}{l}\text { Number } \\
\text { of patients }\end{array}$ \\
\hline Colorectal cancer & 3 \\
Extra-intestinal cancer & 3 \\
Autoimmune diseases, immunosuppression, & 2 \\
glucocorticoids & \\
Prehospital antibiotics for diverticulitis, & 3 \\
symptoms $>5$ days & \\
Other concomitant infection & I \\
Fistula due to diverticulitis & \\
Unremitting symptoms after recent diverticulitis & I \\
Perforation of diverticulum, absence of diverticulitis & I \\
Inflammatory bowel disease & - \\
Liver or severe kidney disease & - \\
Recent surgery & - \\
\hline Notes: ${ }^{\text {} A}$ patient with a colovesical fistula and Hinchey la diverticulitis underwent \\
an urgent operation. Two additional patients without acute inflammation underwent \\
an operation semi-electively for a colovesical fistula (CRP: I mg/L and 3 mg/L). \\
bUlcerative colitis or Crohn's disease. \\
Abbreviation: CRP, C-reactive protein.
\end{tabular}

disorders may increase CRP baseline levels. ${ }^{16}$ The exclusion of all these factors increases the homogeneity of the sample, which is essential for the avoidance of bias in any research correlating CRP levels with CT grading.

In view of the above, diseases or conditions that could potentially affect baseline CRP levels formed the exclusion criteria of this study (Table 2). They comprised the presence or history of extracolonic cancer, autoimmune diseases, inflammatory bowel disease, glucocorticoid therapy, ${ }^{14}$ or any other immunosuppressive state (either due to disease, transplantation, or medication). Accordingly, patients with a concomitant infection of another organ, prehospital antibiotics (either for diverticulitis or another cause), recent failed discharge, symptoms $>5$ days, recent surgery (within 1 month), and liver disease were excluded for the same reason. Furthermore, patients with colorectal cancer documented before the study period, during the hospitalization, or at a follow-up colonoscopy were also not considered for analysis. Lastly, patients with chronic sequelae of $\mathrm{AD}$, such as a colonic fistula or unremitting/recurrent symptoms after a previous episode ${ }^{17}$ and perforation of a diverticulum without underlying inflammation (eg, during colonoscopy), were considered as distinct entities and excluded.

\section{Statistics}

Continuous variables were compared using the Mann-Whitney $U$ test. The association between categorical data was assessed using Fisher's exact test and the chi-squared test. Statistical significance was set at a $P$-value of $<0.05$. Only twotailed, statistically significant results were considered for multivariate analysis. Logistic regression analysis was used for the assessment of independent predictor factors (multivariate analysis). Receiver operating characteristic (ROC) analysis was used to detect the best prognostic cutoff value of the continuous variables, which were found to be independent risk factors for the outcome. The statistical software package used for the analysis was SPSS 21.0.

\section{Results}

Ninety-nine patients with AD were analyzed. Their characteristics and clinical information are given in Table 3. The excluded cases are depicted in Table 2 . In all, 88 cases with a mild disease (Hinchey Ia and Ib) had a median CRP of $80.35 \mathrm{mg} / \mathrm{L}$ (range $1.78-299.58 \mathrm{mg} / \mathrm{L}$ ) and were treated conservatively. Eleven patients with severe CT findings (Hinchey > Ib) had a median CRP of $236 \mathrm{mg} / \mathrm{L}$ (range 166-297 mg/L), and nine of them underwent an invasive procedure (Table 1). The median CRP level for Hinchey II was $196.0 \mathrm{mg} / \mathrm{L}(166-286.85 \mathrm{mg} / \mathrm{L})$, and the median CRP level for Hinchey III/IV was $258.5 \mathrm{mg} / \mathrm{L}$ (201-297 mg/L). An 89-year-old woman with Hinchey II developed bacteremia and died of multi-organ failure (index CRP $259 \mathrm{mg} / \mathrm{L}$ ).

Index CRP $(P<0.001)$, white blood cells (WBCs; $P<0.001)$, neutrophil/lymphocyte $(\mathrm{N} / \mathrm{L})$ ratio $(P<0.001)$, serum creatinine $(P=0.004)$, serum glucose $(P=0.031)$, generalized peritonitis $(P=0.011)$, abdominal tenderness in more than two quadrants $(P=0.009)$, and urinary symptoms $(P=0.026)$ were related to increased radiological severity according to the univariate analysis. Of these factors, only index CRP was an independent predictor for radiological severity in the multivariate logistic regression analysis $(P=0.038)$. ROC curve analysis showed that the best CRP cutoff value for the prediction of Hinchey $>\mathrm{Ib}$ is $173 \mathrm{mg} / \mathrm{L}$ (sensitivity $90.9 \%$, specificity $90.9 \%$, area under the curve [AUC] 95.8, $P<0.001)$. The negative predictive value (NPV) was $98.7 \%$, and the positive predictive value (PPV) was 55.5\%. The relation between the index CRP values of this series and radiological severity is depicted in Figure 1.

There were 61 (61.6\%) patients with CRP levels $<100 \mathrm{mg} / \mathrm{L}$. All of them had a Hinchey radiological grade of I (sensitivity $100 \%$, specificity $68.2 \%$ ), and none necessitated any intervention during the acute attack. In all, 25 (25.3\%) patients had CRP levels $<50 \mathrm{mg} / \mathrm{L}$ (sensitivity $100 \%$, specificity $28.4 \%$ ). All patients who underwent CT drainage or operation had CRP levels $>173 \mathrm{mg} / \mathrm{L}$.

The Hinchey stage of the excluded cases ranged between Ia and III, with index CRP values varying between 1 and $115 \mathrm{mg} / \mathrm{L}$. Of these cases, two patients received the Hartmann 
Table 3 Patient characteristics, clinical information, and outcome

\begin{tabular}{|c|c|c|c|c|}
\hline Variables & $\begin{array}{l}\text { Total } \\
(\mathrm{N}=99)\end{array}$ & $\begin{array}{l}\text { Hinchey la/lb } \\
(n=88)\end{array}$ & $\begin{array}{l}\text { Hinchey II/III/IV } \\
(n=I I)\end{array}$ & $P$-value \\
\hline Age (years) & $58.0(30-89)$ & $58(32-83)$ & $56(30-89)$ & 0.832 \\
\hline Male gender & $61(61.6)$ & $55(62.5)$ & $6(54.5)$ & 0.745 \\
\hline Nausea/vomitus & $25(25.3)$ & $23(26.1)$ & $2(18.2)$ & 0.725 \\
\hline Constipation & $16(16.2)$ & $14(15.9)$ & $2(18.2)$ & 1.000 \\
\hline Bloating & $27(27.3)$ & $21(23.9)$ & $6(54.5)$ & 0.065 \\
\hline Loose stools & $23(23.2)$ & $20(22.7)$ & $3(27.3)$ & 0.714 \\
\hline Mucus in stools & $7(7.1)$ & $7(8.0)$ & 0 & 1.000 \\
\hline Blood per rectum & $3(3.0)$ & $3(3.4)$ & 0 & 1.000 \\
\hline Urinary symptoms & $18(18.2)$ & $13(14.8)$ & $5(45.5)$ & 0.026 \\
\hline Abdominal tenderness $>2$ quadrants & $5(5.1)$ & $2(2.3)$ & $3(27.3)$ & 0.009 \\
\hline Generalized peritonitis & $2(2.0)$ & 0 & $2(18.2)$ & 0.011 \\
\hline Rebound tenderness & $4 I(4 I .4)$ & $35(39.8)$ & $6(54.5)$ & 0.518 \\
\hline Palpable mass & $7(7.1)$ & $5(5.7)$ & $2(18.2)$ & 0.173 \\
\hline Hemodynamic instability & $\mathrm{I}(\mathrm{I} .0)$ & 0 & I (9.1) & 0.111 \\
\hline Diabetes & $18(18.2)$ & $16(18.2)$ & $2(18.2)$ & 0.681 \\
\hline Fever $\left({ }^{\circ} \mathrm{C}\right)$ & $37.6(36.0-39.8)$ & $37.55(36-39.5)$ & $38.1(36.3-39.8)$ & 0.153 \\
\hline WBC $\left(10^{9} / L\right)$ & I $3.6(4.78-28.2)$ & $13.18(4.78-22.6)$ & I7.| (|2.49-28.2) & $<0.001$ \\
\hline $\mathrm{N} / \mathrm{L}(\%)$ & $5.55(1.59-38.33)$ & $5.32(1.59-38.33)$ & 14.17 (6.79-18.2) & $<0.001$ \\
\hline CRP (mg/L) & 86.74 (1.78-299.58) & 80.35 (1.78-299.58) & $236.0(166-297)$ & $<0.001$ \\
\hline CRP $>173(\mathrm{mg} / \mathrm{L})$ & $18(18.2)$ & $8(9.1)$ & $10(90.9)$ & $<0.001$ \\
\hline Serum creatinine $(\mathrm{mg} / \mathrm{dL})$ & $\mathrm{I} .0(0.5-1.8)$ & $0.9(0.5-1.7)$ & $1.2(0.8-1.8)$ & 0.004 \\
\hline Serum glucose (mg/dL) & $106(73-275)$ & $104(73-275)$ & $118(92-149)$ & 0.031 \\
\hline Known diverticulosis & $38(38.4)$ & $34(38.6)$ & $4(36.4)$ & 1.000 \\
\hline Recurrent attack & $30(30.3)$ & $27(30.7)$ & $3(27.3)$ & 1.000 \\
\hline Number of previous attacks & $0.0(0-5)$ & $0.0(0-3)$ & $0.0(0-5)$ & 0.509 \\
\hline In-hospital days & $4(0-31)$ & $3(0-10)$ & $13(5-3 \mid)$ & $<0.001$ \\
\hline Percutaneous drainage/operation & $9(10)$ & 0 & $9(81.8)$ & $<0.001$ \\
\hline Death & $\mathrm{I}(\mathrm{I} .0)$ & 0 & $\mathrm{I}(9.1)$ & 0.111 \\
\hline
\end{tabular}

Notes: Clinical status, laboratory results, and imaging obtained at the initial presentation of the patients at the emergency unit. Continuous data are presented as median (range) and categorical data as $\mathrm{n}(\%)$.

Abbreviations: WBC, white blood cells; N/L, neutrophil/lymphocyte ratio; CRP, C-reactive protein.

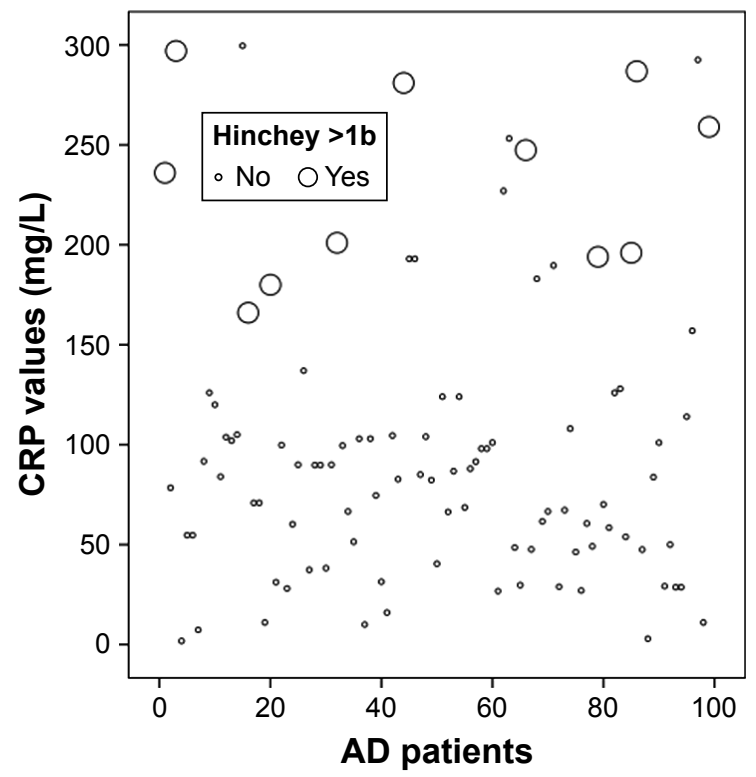

Figure I Scatter plot showing the distribution of CRP values for cases with Hinchey $\mathrm{la}$ and $\mathrm{lb}$ (small circles) and for cases with Hinchey $>\mathrm{lb}$ (larger circles).

Abbreviations: CRP, C-reactive protein; $A D$, acute diverticulitis. procedure: one had Hinchey Ia and an index CRP of $115 \mathrm{mg} / \mathrm{L}$ and the other had Hinchey III with an index CRP of $106 \mathrm{mg} / \mathrm{L}$. A patient with Hinchey II and an index CRP of $64 \mathrm{mg} / \mathrm{L}$ received percutaneous drainage.

\section{Discussion}

This study showed that a high index CRP value is independently related to the increased severity of $\mathrm{AD}$. A CRP level $>173 \mathrm{mg} / \mathrm{L}$ at presentation accurately identifies patients with major acute complications that correspond to Hinchey stages $>\mathrm{Ib}$. Patients with the mere phlegmonous disease (Hinchey Ia) or a minor perforation or small abscess (Hinchey Ib) presented with lower index CRP values and were all treated medically in our series. The abovementioned results apply to patients without comorbidities or concomitant conditions that affect CRP and patients without chronic sequelae from previous diverticulitis. Univariate analysis showed the association between various further parameters 
(obtained during the initial evaluation of the patients) and severe AD. These included clinical signs of widespread infection within the abdomen, an increased N/L ratio and $\mathrm{WBCs}$, and laboratory measurements that may be deranged during acute infection (serum creatinine and glucose). However, these factors were not identified as independent predictive factors for severe disease.

Our findings highlight the importance of obtaining a CT scan in patients with suspected AD and an index CRP $>173 \mathrm{mg} / \mathrm{L}$. Routine measurement of index CRP is essential, especially when the diagnosis has been reached clinically or with ultrasound. ${ }^{21}$ Diagnosis with clinical-only means has been reported in part of the patients of series from dedicated centers, ${ }^{2,18,20,24}$ and its accuracy ranges from $43 \%$ to $68 \% .^{21}$ Index CRP is particularly important in rural or remote areas with limited access to diagnostic imaging. ${ }^{25,26}$

Two previous investigations assessed the role of initial CRP in the prediction of the clinical outcome during index hospitalization. A small prospective observational study reported that patients who underwent emergency surgery for $\mathrm{AD}$ had higher index CRP levels (281 vs $58 \mathrm{mg} / \mathrm{L}){ }^{22}$ Similarly, a retrospective study showed that a CRP value of $>170 \mathrm{mg} / \mathrm{L}$ (sensitivity $87.5 \%$, specificity $91.1 \%$ ) is associated with the need for percutaneous drainage and/or surgery in a cohort with the same exclusion criteria as the present study. ${ }^{19}$

So far, only retrospective reports have correlated CRP with radiological grading. ${ }^{3,12-14}$ Attention should be paid to the fact that these investigations classified minor perforations and small abscesses (Hinchey Ib) as "severe disease" alongside larger abscesses and generalized peritonitis (Hinchey $>\mathrm{Ib}$ ). This is also a useful distinction, but it is not entirely consistent with the current clinical perspective, according to which perforated cases with minor radiological complications (Hinchey $\mathrm{Ib}$ ) are treated medically during the acute phase. In addition, labeling patients who are as a rule managed conservatively (Hinchey $\mathrm{Ib})^{4-9}$ as having severe disease may contribute to lower sensitivity rates for CRP's predictive value. . $^{3,12-14}$

The earliest of these reports found that local perforation was unlikely for a CRP value of $<50 \mathrm{mg} / \mathrm{L}$, whereas a value of $>200 \mathrm{mg} / \mathrm{L}$ was a strong indicator of either minor or major complications in imaging (Hinchey $>\mathrm{Ia}) .{ }^{12}$ However, it should be noted that CRP values were measured during a 48-hour period before CT. ${ }^{12}$ This may have created a degree of bias, as CRP levels are highly time dependent and display significant changes in sub-24-hour intervals. ${ }^{23}$ Another study noted that index CRP levels were significantly lower in patients using corticosteroids for the same radiological degree of severity in $\mathrm{AD}$ and provided a rather low CRP cutoff level (CRP >90 mg/L, 88\% sensitivity and 75\% specificity) for the prediction of Hinchey $>$ Ia in the entire cohort. ${ }^{14}$ The authors stated that $\sim 20 \%$ of CT scans could have been avoided with the use of a CRP threshold of $<50 \mathrm{mg} / \mathrm{L} .{ }^{14}$ Two further investigations provided CRP cutoff values of $150 \mathrm{mg} / \mathrm{L}$ (sensitivity $65 \%$, specificity $85 \%)^{3}$ and $175 \mathrm{mg} / \mathrm{L}$ (sensitivity $61 \%$, specificity $82 \%$ ) $^{13}$ for the prediction of Hinchey $>$ Ia. The studies mentioned earlier evaluated unselected cases, as prehospital antibiotics, corticosteroids, immunosuppression, or comorbidities and conditions that affect CRP basal levels were not excluded. ${ }^{3,12-14}$ This may further explain the calculation of lower sensitivity rates for the CRP cutoff level. ${ }^{3,12,13}$

We used prospectively collected data, which increased the strength of our investigation. Nevertheless, a future study with a larger cohort is necessary to provide definitive conclusions through the evaluation of a larger subgroup with radiologically severe disease. The present study supported a more clinically oriented definition of severity in patients with AD: local perforation or small abscess (Hinchey Ib) was considered as an uncomplicated disease resulting in the improved NPV of the CRP cutoff level, whereas major perforation only (Hinchey $>\mathrm{Ib}$ ) constituted the complicated disease. Indeed, patients with the perforated disease who commonly necessitate a higher level of care and interventional or operative management are those with Hinchey $>\mathrm{Ib} .{ }^{15}$ Treatment with antibiotics (and in a few cases, even observation without antibiotics) is generally successful for the resolution of the acute event in Hinchey Ib patients. ${ }^{4-9}$ Sensitivity and specificity rates were strengthened by the use of dedicated exclusion criteria, which were specifically tailored to the physiology of CRP. The inclusion of patients with factors affecting baseline index CRP levels in the analysis would have decreased the accuracy of the CRP cutoff value, as may have happened in previous studies. Indeed, excluded patients who received interventions in our series had index CRP levels between 64 and $115 \mathrm{mg} / \mathrm{L}$. As a result, index CRP should be used as a predictor of the severity of AD in properly selected patients.

As our patients had undergone a CT scan, this investigation is not designed to support the argument that the amount of staging CT should be reduced in patients with AD and low index CRP values. Since the great majority of patients with an acute episode of the disease experience an uneventful clinical course, ${ }^{1-3}$ dedicated future research should aim to identify 
how to avoid unnecessary CT scans. The role of index CRP warrants further evaluation in this context, taking into consideration that clinical $1^{2,18,20,24}$ and/or ultrasound ${ }^{21,27}$ diagnosis is gaining increasing popularity. It is nonetheless judicious that patients with adverse characteristics such as older age, ${ }^{3}$ bowel obstruction, significant comorbidity or immunosuppressive medication, ${ }^{20}$ organ dysfunction, ${ }^{24}$ systemic sepsis, and hypotension at presentation should undergo a CT scan even if they have a low index CRP value. Lastly, it should be mentioned that the maximum abscess diameter in Hinchey $\mathrm{Ib}$ cases in our series was $3 \mathrm{~cm}$. Therefore, conclusions cannot be drawn for Hinchey $\mathrm{Ib}$ patients with a local pericolic or mesocolic abscess $>3 \mathrm{~cm}$.

\section{Conclusion}

A high index CRP in patients with $\mathrm{AD}$ predicts severe complications, defined as an abscess $\geq 3 \mathrm{~cm}$ or findings of peritonitis in the CT scan. CRP should not be used as a predictor of severity if there are concomitant conditions that may affect its baseline levels. Our investigation calls for the use of meticulous methodological criteria in future research, including patient selection and a clinically oriented definition of disease severity.

\section{Disclosure}

The authors report no conflicts of interest in this work.

\section{References}

1. Bharucha AE, Parthasarathy G, Ditah I, et al. Temporal Trends in the Incidence and Natural History of Diverticulitis: A Population-Based Study. Am J Gastroenterol. 2015;110(11):1589-1596.

2. Jamal Talabani A, Lydersen S, Endreseth BH, Edna TH. Major increase in admission- and incidence rates of acute colonic diverticulitis. Int $J$ Colorectal Dis. 2014;29(8):937-945.

3. Mäkelä JT, Klintrup K, Takala H, Rautio T. The role of C-reactive protein in prediction of the severity of acute diverticulitis in an emergency unit. Scand J Gastroenterol. 2015;50(5):536-541.

4. Mali JP, Mentula PJ, Leppäniemi AK, Sallinen VJ. Symptomatic Treatment for Uncomplicated Acute Diverticulitis: A Prospective Cohort Study. Dis Colon Rectum. 2016;59(6):529-534.

5. Dharmarajan S, Hunt SR, Birnbaum EH, Fleshman JW, Mutch MG. The efficacy of nonoperative management of acute complicated diverticulitis. Dis Colon Rectum. 2011;54(6):663-671.

6. Gregersen R, Mortensen LQ, Burcharth J, Pommergaard HC, Rosenberg J. Treatment of patients with acute colonic diverticulitis complicated by abscess formation: A systematic review. Int J Surg. 2016;35:201-208.

7. Thorisson A, Smedh K, Torkzad MR, Påhlman L, Chabok A. CT imaging for prediction of complications and recurrence in acute uncomplicated diverticulitis. Int J Colorectal Dis. 2016;31(2):451-457.
8. Sallinen VJ, Mentula PJ, Leppäniemi AK. Nonoperative management of perforated diverticulitis with extraluminal air is safe and effective in selected patients. Dis Colon Rectum. 2014;57(7):875-881.

9. Daniels L, Ünlü Ç, de Korte N, et al. Randomized clinical trial of observational versus antibiotic treatment for a first episode of CT-proven uncomplicated acute diverticulitis. Br J Surg. 2017;104(1):52-61.

10. Sartelli M, Catena F, Ansaloni L, et al. WSES Guidelines for the management of acute left sided colonic diverticulitis in the emergency setting. World J Emerg Surg. 2016;11:37.

11. Costi R, Cauchy F, Le Bian A, et al. Challenging a classic myth: pneumoperitoneum associated with acute diverticulitis is not an indication for open or laparoscopic emergency surgery in hemodynamically stable patients. A 10-year experience with a nonoperative treatment. Surg Endosc. 2012;26(7):2061-2071.

12. Käser SA, Fankhauser G, Glauser PM, Toia D, Maurer CA. Diagnostic value of inflammation markers in predicting perforation in acute sigmoid diverticulitis. World J Surg. 2010;34(11):2717-2722.

13. van de Wall BJ, Draaisma WA, van der Kaaij RT, et al. The value of inflammation markers and body temperature in acute diverticulitis. Colorectal Dis. 2013;15(5):621-626.

14. Nizri E, Spring S, Ben-Yehuda A, et al. C-reactive protein as a marker of complicated diverticulitis in patients on anti-inflammatory medications. Tech Coloproctol. 2014;18(2):145-149.

15. Feingold D, Steele SR, Lee S, et al. Practice parameters for the treatment of sigmoid diverticulitis. Dis Colon Rectum. 2014;57(3):284-294.

16. Montecucco F, Mach F. New evidences for C-reactive protein (CRP) deposits in the arterial intima as a cardiovascular risk factor. Clin Interv Aging. 2008;3(2):341-349.

17. Ambrosetti P, Gervaz P. Management of sigmoid diverticulitis: an update. Updates Surg. 2016;68(1):25-35.

18. Ridgway PF, Latif A, Shabbir J, et al. Randomized controlled trial of oral vs intravenous therapy for the clinically diagnosed acute uncomplicated diverticulitis. Colorectal Dis. 2009;11(9):941-946.

19. Kechagias A, Rautio T, Kechagias G, Mäkelä J. The role of C-reactive protein in the prediction of the clinical severity of acute diverticulitis. Am Surg. 2014;80(4):391-395.

20. Lorimer JW, Doumit G. Comorbidity is a major determinant of severity in acute diverticulitis. Am J Surg. 2007;193(6):681-685.

21. Andeweg CS, Mulder IM, Felt-Bersma RJF, et al. Guidelines of Diagnostics and Treatment of Acute Left-Sided Colonic Diverticulitis. Dig Surg. 2013;30(4-6):278-292.

22. John SK, Teo NB, Forster AL. A prospective study of acute admissions in a surgical unit due to diverticular disease. Dig Surg. 2007;24(3): 186-190.

23. Kechagias A, Rautio T, Mäkelä J. The early C-reactive protein trend does not have a role in monitoring acute diverticulitis progression. Chir. 2016;111:43-47.

24. Sallinen VJ, Leppäniemi AK, Mentula PJ. Staging of acute diverticulitis based on clinical, radiologic, and physiologic parameters. J Trauma Acute Care Surg. 2015;78(3):543-551.

25. Nixon G, Samaranayaka A, de Graaf B, et al. Geographic disparities in the utilisation of computed tomography scanning services in southern New Zealand. Health Policy. 2014;118(2):222-228.

26. Lysdahl KB, Børretzen I. Geographical variation in radiological services: a nationwide survey. BMC Health Serv Res. 2007;7:21.

27. de Korte N, Kuyvenhoven JP, van der Peet DL, et al. Mild colonic diverticulitis can be treated without antibiotics. A case-control study. Colorectal Dis. 2012;14(3):325-330.

28. Wasvary H, Turfah F, Kadro O, Beauregard W. Same hospitalization resection for acute diverticulitis. Am Surg. 1999;65(7):632-635. 
Therapeutics and Clinical Risk Management

Dovepress

\section{Publish your work in this journal}

Therapeutics and Clinical Risk Management is an international, peerreviewed journal of clinical therapeutics and risk management, focusing on concise rapid reporting of clinical studies in all therapeutic areas outcomes, safety, and programs for the effective, safe, and sustained use of medicines. This journal is indexed on PubMed Central, CAS,
EMBase, Scopus and the Elsevier Bibliographic databases. The manuscript management system is completely online and includes a very quick and fair peer-review system, which is all easy to use. Visit http://www.dovepress.com/testimonials.php to read real quotes from published authors.

Submit your manuscript here: http://www.dovepress.com/therapeutics-and-clinical-risk-management-journal 